\title{
Design and Validation of a Probe for Spatially and Temporally Resolved Measurements of Vorticity and Strain Rates in Compressible Turbulence Interactions
}

\begin{abstract}
S. Xanthos, M. Gong, Y. Andreopoulos
A custom-made hot-wire vorticity probe was designed and developed capable of measuring the time-dependent highly fluctuating three dimensional velocity and vorticity vectors, and associated total temperature, in non-isothermal and inhomogeneous flows with reasonable spatial and temporal resolution. These measurements allowed computation of the vorticity stretching/tilting terms, vorticity generation through dilatation terms, full dissipation rate of the kinetic energy term and full rate-of-strain tensor. The probe has been validated experimentally in low-speed boundary layers and used in the CCNY Shock Tube Research Facility, where interactions of planar expansion waves or shock waves with homogeneous and isotropic turbulence have been investigated at several Reynolds numbers.
\end{abstract}

Keywords: vorticity measurements, compressible turbulence, shock and expansion waves.

\section{Nomenclature}

C Speed of Sound

Cp Heat capacity

E Probe voltage output

$m \quad$ Mass

$M \quad$ Mesh Size

$P \quad$ Pressure

$R \quad$ Gas constant

Re Reynolds number

$S \quad$ Probe sensitivity

$S_{i j} \quad$ Strain Rate

$T$ Temperature

$T_{0} \quad$ Total temperature

$T_{\mathrm{r}} \quad$ Reference temperature

$T_{\mathrm{w}} \quad$ Wire temperature

$U_{i} \quad$ Instantaneous Velocity

$u_{i} \quad$ Velocity fluctuation

$\bar{U}_{i} \quad$ Mean Velocity

$X \quad$ Distance from grid to probe

$x_{i} \quad$ Coordinates

$\varepsilon \quad$ Dissipation rate

$\eta \quad$ Kolmogorov's microscale

$\lambda \quad$ Taylor's microscale

$v \quad$ Viscosity

$\rho$

\section{Introduction}

Vorticity is a quantity that can describe viscous effects in a flow field much better than velocity, and it is very well suited for defining and identifying organized structures in time-dependent vortical flows because the streamlines and pathlines are completely different in two different inertial frames of reference. In this respect, better understanding of the nature of turbulent structures and vortical motions of turbulent flows, particularly in the high-wavenumber region, often requires spatially and temporally resolved measurements of velocity derivatives.

Lighthill [1] in his wide-ranging introduction to boundary-layer theory provided an extensive description of vorticity dynamics in a variety of flows by using vorticity as a primitive variable for various theoretical considerations. In the past six years our group has made an intense effort to extend the successful techniques developed for measurements of three-dimensional vorticity and the rate-of-strain tensor. These techniques involve measurements in low-speed turbulent boundary layers of Reynolds numbers $\operatorname{Re}_{\theta}=2,700$, Ref [2-5], in vortices generated over delta wings [6], compressible flows with shock interactions [7-9] and recently compressible flows with expansion wave interactions. As mentioned by Vukoslavcevic et al. [10] and Wallace and Foss [11], the measurement of vorticity was elusive until a few years ago, when this effort was first undertaken.

In the present work, details of the design and evaluation of a vorticity probe based on hot-wire anemometry are presented. Some of the fundamental aspects of turbulence can be studied better in flow configurations where the flow is nearly homogeneous and turbulence is nearly isotropic. The presence of a solid wall as a boundary in turbulent flows complicates its understanding by introducing large mean velocity gradients at the wall which are responsible for the continuous production of turbulence. Better understanding of the effects of a shock wave or expansion waves on turbulence can be obtained by considering their interaction with grid-generated turbulence where no streamline curvature and a wall with no-slip conditions are present. The flow behind a turbulence-generating grid contains a large variety of turbulent scales, the size of which depends on the distance from the grid and the grid mesh size. 
The custom-made vorticity probe has been used for measurements in the interaction of isotropic and homogeneous turbulence with expansion waves. In all experiments, the main objective has been to better understand the physics of the interactions and to establishing the behavior of the vorticity field. Detailed experimental investigations of interactions of expansion waves with isotropic turbulence simply do not exist. The only existing studies are rather limited and have been confined to turbulent boundary layers, as will be discussed shortly.

\section{Experimental set-up and techniques}

The interactions have been simulated experimentally in the CCNY Shock Tube Facility shown in Fig. 1. The shock tube facility is of large-scale dimensions with an inside diameter of 12 inches $(304 \mathrm{~mm})$ and total length of 90 feet $(27.4 \mathrm{~m})$, including all components.

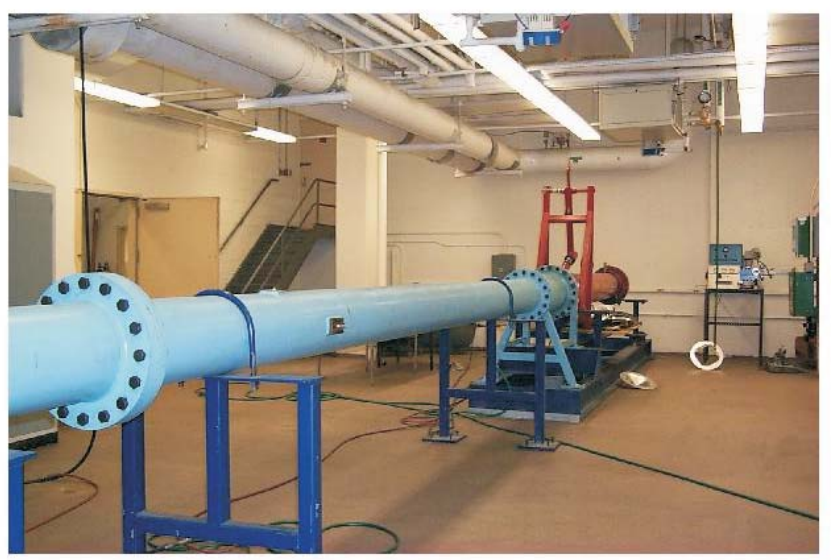

Fig. 1: Shock tube facility at CCNY, Driver section

The present shock tube facility has three distinguishing features. The most significant one is the ability to control the strength of the reflected shock and the flow quality behind it by using a removable porous end wall, placed at the flange between the dump tank and the working section. The impact of the shock wave on the end wall would result in a full normal shock reflection in case of zero porosity (solid wall), a weak shock reflection in case of moderate porosity, or expansion waves in case of unit porosity (open end wall). The second feature of the facility is the ability to vary the total length of the driven section by adding or removing one of several pieces or modules that are available, or to rearrange their layout. Proper arrangement of the layout of the various modules of the shock tube can maximize the duration of the useful flow. The third feature of the facility is its large diameter, which allows for a large area of uniform flow in the absence of wall effects thus providing a platform for high spatial resolution turbulence measurements.

A turbulence-generating grid installed at the beginning of the working section of the facility was used to generate a homogeneous and isotropic turbulent flow. The interactions of this flow with shock waves was produced by using the porous end wall of the shock tube, while an open end wall was used to generate an expansion wave. Fig. 2 shows schematically the interaction with expansion waves (EW).

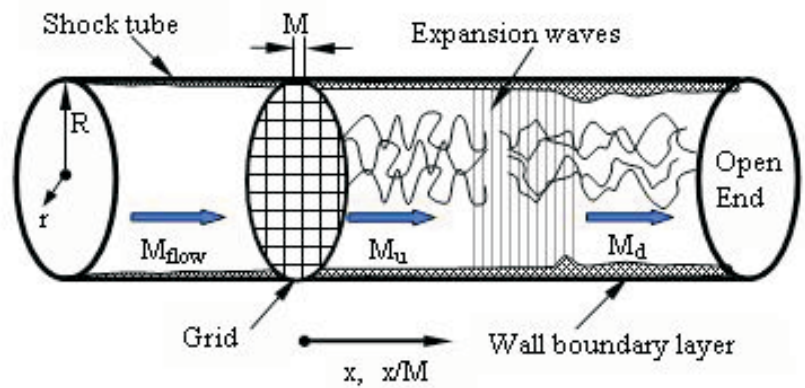

Fig. 2: Schematic of flow interaction with expansion waves

The working (test) section is fitted with several hot-wire and pressure ports, (see Fig. 3 for working section with visible wall pressure taps and grid). Thus pressure, velocity and temperature data can be acquired simultaneously at various locations downstream from the grid, and therefore we can reduce the variance between measurements. High-frequency pressure transducers and hot wire anemometry have been used in the present investigation. Pressure transducers were placed throughout the driven section in order to monitor the passage of the shock wave and also to check its uniformity through the driven section. For the present experiments of velocity and vorticity measurements, high frequency response Kulite pressure transducers type XCQ-062 were installed in the shock tube at multiple locations, so that wall pressure can be measured simultaneously as a function of time.

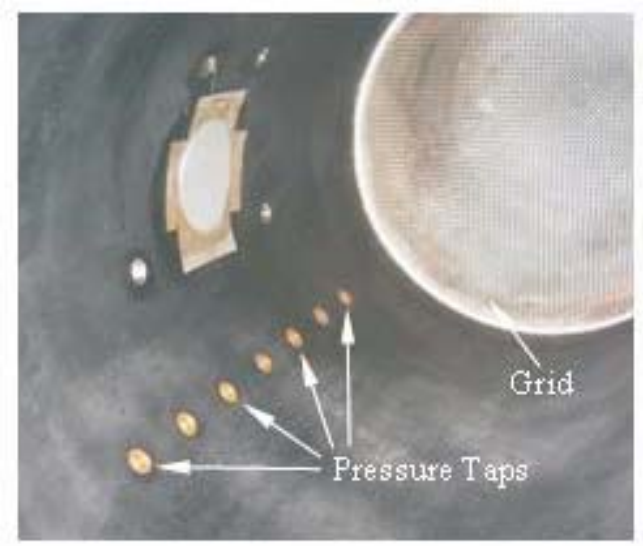

Fig. 3: Working section with visible pressure taps and turbulence generating grid

Time-dependent, three-dimensional vorticity measurements were carried out by using the new vorticity probe, $[4,5$, $8,13]$. Fig. 4 shows a layout of the arrangement for the simultaneous measurements of vorticity and wall pressure along the length of the working section.

The shock tube was pressurized so that leaks could be detected, as well as to calibrate the pressure transducers. The shock tube was free of leaks and the static response of the transducers was found to be linear. Aluminum plates were used as diaphragms and were placed in between the driver and the driven initially conically shaped section. A detailed description of the facility and the results of the qualification tests can be found in the work by Briassulis [12], Briassulis et al. [9] and Agui et al. [13]. All data were digitized by 4 National Instruments Analog-to Digital-Converters model 


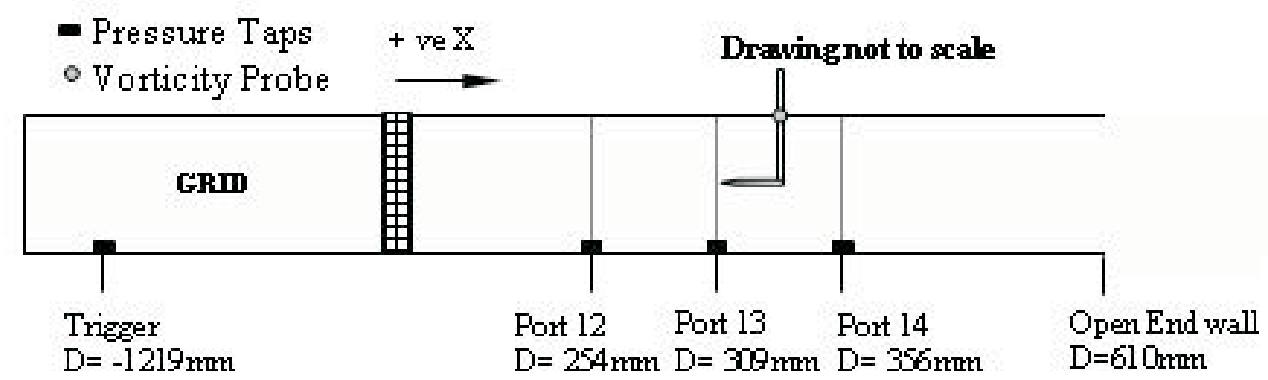

Fig. 4: 2-D schematic of the working section with pressure and vorticity probe locations along the $2 \mathrm{ft}$ length

NI PCI 6120 with 16 bit resolution and 1Mhz per channel for a total of 16 channels. The data acquisition system was triggered by the arrival of the shock wave at the location of a wall pressure transducer upstream of the grid. The grid was installed in the beginning of the working section, as shown in Figs. 3 and 4.

\section{Vorticity measurements}

A new multi hot-wire probe has been developed which is capable of measuring velocity-gradient related quantities in non-isothermal flows or in compressible flows. The present probe has been built using the experience gained with vorticity measurements in incompressible flows [4] by using a probe with nine wires, and with velocity measurements in compressible flows by using single and cross-wire probes [9, 14]. The present vorticity probe, which consists of 12 wires, is a modification of the original design with nine wires [4]. The three additional wires were operated in the so-called Constant Current Mode and used to measure time-dependent total temperature.

Since the probe essentially consists of a set of three modules or arrays it is necessary to provide several key features of the individual hot-wire modules (see Figs. 5a and 5b). Each module contains three hot-wires operated in the Constant Temperature Mode (CTM) and one cold-wire sensor operated in the Constant Current Mode (CCM). Each wire of the triple wire sub-module is mutually orthogonal to each other, thus oriented at 54.7 degrees to the probe axis. Each of the $5 \mu \mathrm{m}$ diameter tungsten sensors is welded on two individual prongs, which have been tapered at the tips. Each sensor is operated independently hence no common prongs are used. Each of the $2.5 \mu \mathrm{m}$ diameter cold-wire was located on the outer part of the sub-module. Extensive testing of the probe has been carried out to assess its performance in shock tube flows. The reader is referred to the work of Briassulis et al. [8] and Agui et al. [9] for details of the tests and the techniques associated with the use of the probe. The probe was also tested in low-speed incompressible boundary layer flows, where vorticity measurements have been obtained in the past with a 9-wire probe [4] and with optical techniques [15]. Comparison of the data obtained with the new probe with these previous measurements was very satisfactory. The cold-wire signals were first converted to total temperature, which together with the hot wire signals were used to obtain instantaneous three-dimensional mass fluxes at three neighboring locations within the probe. The numerical techniques and algorithms used in the computations of velocity gradients were very similar to those described by Honkan \& Andreopoulos [4]. The only difference is that in the present case mass fluxes and their gradients were computed at the centroid of each module instead of velocities and velocity gradients. Mass fluxes were further separated into density and velocity by using the method adopted by Briassulis et al. [12]. Decoupling density from mass fluxes assumes that the static pressure fluctuations are small. This is the so-called "weak" version of the original "strong Reynolds analogy" hypothesis of Morkovin. The original hypothesis is based on the assumption that pressure and total temperature fluctuations are very small. In the present work, total temperature was measured directly and therefore no corresponding assumptions were needed. The pressure, however, was measured at the wall beneath the hot wire probes and not at the location of the hot wire measurement inside the flow field. The mean value of this pressure signal was used to separate the density and velocity signals, since no mean pressure variation has been
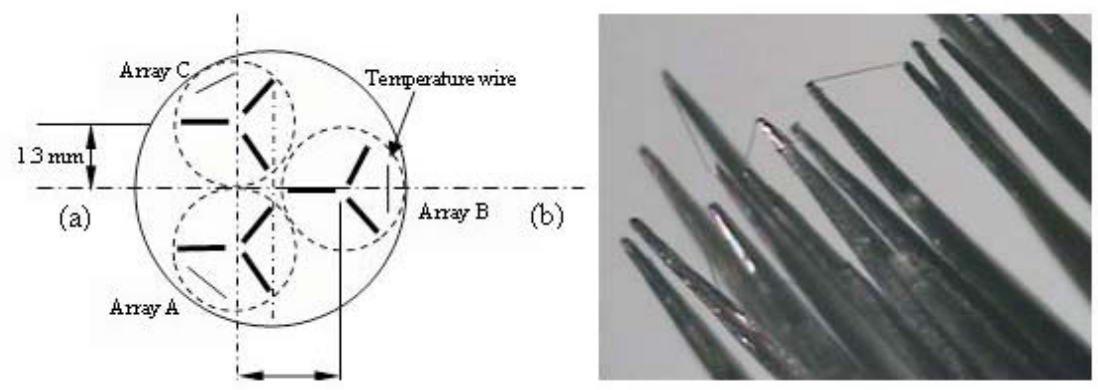

Fig. 5: Vorticity probe: (a) probe sensor geometry and arrays, (b) close-up view of the probe 
detected across a given section of the flow. The procedure involves an expression for mass flux, mi, in terms of total temperature, $T_{0}$, and pressure, $p$, at the centroid of each array:

$$
m_{i}=\rho U_{i}=\frac{p U_{i}}{R T}=\frac{p U_{i}}{\left[R\left(T_{0}-\frac{U_{k} U_{k}}{2 c_{p}}\right)\right]},
$$

where $U_{i}$ is the instantaneous velocity component, $i=1$, 2 or 3 and $U_{k} U_{k}=U_{1}^{2}+U_{2}^{2}+U_{3}^{2}$. The velocity can be decomposed into

$$
U_{i}=\bar{U}_{i}+u_{i} .
$$

An iterative scheme was used to decouple density and velocity. During the first iteration it was assumed that the quantity $\left(u_{2}^{2}+u_{3}^{2}\right) / 2 c_{p}$, where $u_{2}$ and $u_{3}$ are the velocity components in the spanwise and normal directions respectively, is substantially smaller that the quantity $T_{0}-U_{1}^{2} / 2 c_{p}$. Then the above relation can be rearranged to obtain a quadratic equation for $U_{i}$,

$$
\frac{R m_{i}}{2 c p} U_{i}^{2}+p U_{i}-m_{i} R T_{0}=0 .
$$

For each digitized point, $T_{0}$ and $m_{i}$ were available instantaneously at the centroid of each module while pressure was measured at the wall. If the thin shear layer approximation is invoked, then the pressure at the centroid of the array, which appears in the last equation, can be substituted by the mean pressure at the wall. This assumption is justified because the lateral pressure fluctuations are extremely small and therefore their impact on velocity fluctuations is minimal. The discriminant of the above equation

$$
\Delta=p^{2}+2 m_{i}^{2} R^{2} \frac{T_{0}}{c_{p}}
$$

is always positive and therefore there are two real roots. The product of the two roots, as expressed by the ratio of the last term of the l.h.s. of the quadratic equation to the coefficient of the first term, is always negative. Therefore one root is positive and one negative. The negative root is unrealistic and only the positive root was accepted. The longitudinal velocity component $U_{1}$ was computed first while the other two components were obtained from the mass flux ratios as

$$
u_{2}=\frac{m_{2}}{m_{1} U_{1}} \text { and } u_{3}=\frac{m_{3}}{m_{1} U_{1}} .
$$

These values provided the first estimate of the velocity components which were used to obtain a better estimate of the $U_{k}^{2} / 2 c_{p}$, which subsequently was used to improve the estimate of the velocity components. This iterative scheme required no more than two iterations for convergence.

In summary, it should be emphasized that the major contribution of the present hot wire techniques is the addition of temperature wires to obtain instantaneous information on total temperature. This allowed decoupling of all partial sensitivities of the probe from each other. Thus,

$$
S_{\rho}=\frac{\partial E}{\partial \rho} \neq S_{\rho u}=\frac{\partial E}{\partial \rho u} \neq S_{u}=\frac{\partial E}{\partial u},
$$

where $E$ is the voltage output from the probe. The streamwise derivatives of the three velocity components usually require the use of Taylor's hypothesis of "frozen" convected turbulence to convert temporal derivatives of velocity into spatial derivatives. In the present work this has been accomplished by considering the use of the full momentum equations to estimate the streamwise derivatives of the three velocity components by ignoring the viscous terms. These expressions can be written as:

$$
\begin{aligned}
& \frac{\partial U_{1}}{\partial x_{1}}=-\frac{1}{U_{1}}\left[\frac{\partial U_{1}}{\partial t}+U_{2} \frac{\partial U_{1}}{\partial x_{2}}+U_{3} \frac{\partial U_{1}}{\partial x_{3}}+\frac{1}{\rho} \frac{\partial p}{\partial x_{1}}\right], \\
& \frac{\partial U_{2}}{\partial x_{1}}=-\frac{1}{U_{1}}\left[\frac{\partial U_{2}}{\partial t}+U_{2} \frac{\partial U_{2}}{\partial x_{2}}+U_{3} \frac{\partial U_{2}}{\partial x_{3}}+\frac{1}{\rho} \frac{\partial p}{\partial x_{2}}\right], \\
& \frac{\partial U_{3}}{\partial x_{1}}=-\frac{1}{U_{1}}\left[\frac{\partial U_{3}}{\partial t}+U_{2} \frac{\partial U_{3}}{\partial x_{2}}+U_{3} \frac{\partial U_{3}}{\partial x_{3}}+\frac{1}{\rho} \frac{\partial p}{\partial x_{3}}\right] .
\end{aligned}
$$

Thus the determination of the streamwise gradients $\partial U_{i} / \partial x_{1}$ is not based entirely on Taylor's original hypothesis. All the terms in the above are available at each time step with

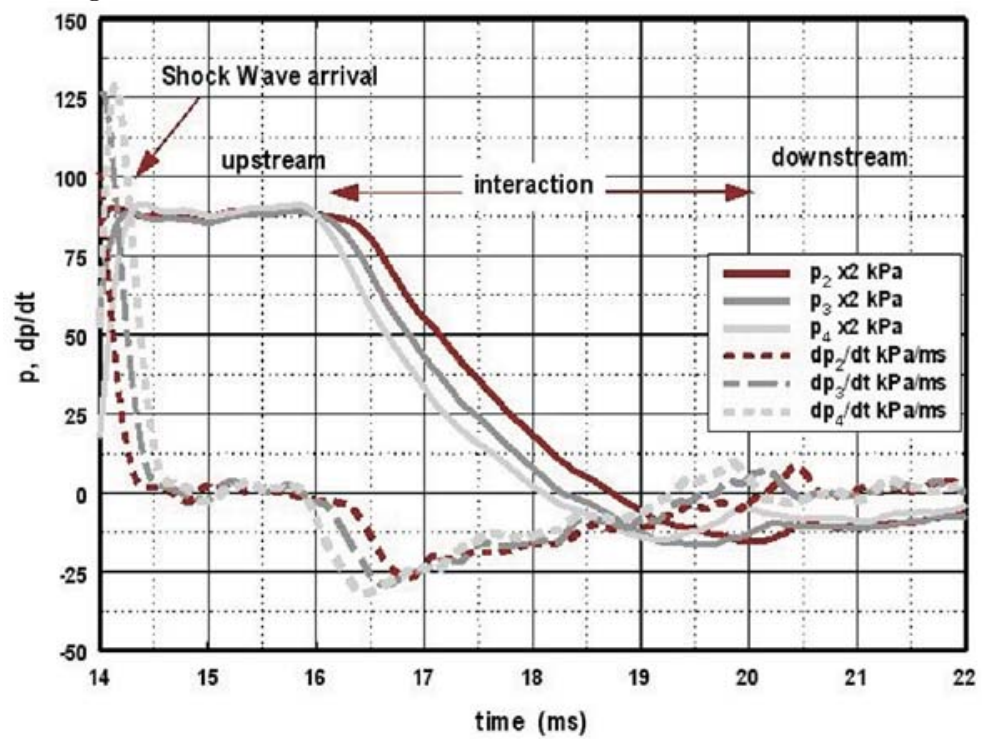

Fig. 6: Typical time dependent wall pressure signals and their temporal gradients at three different locations in the working section below hot probes. 


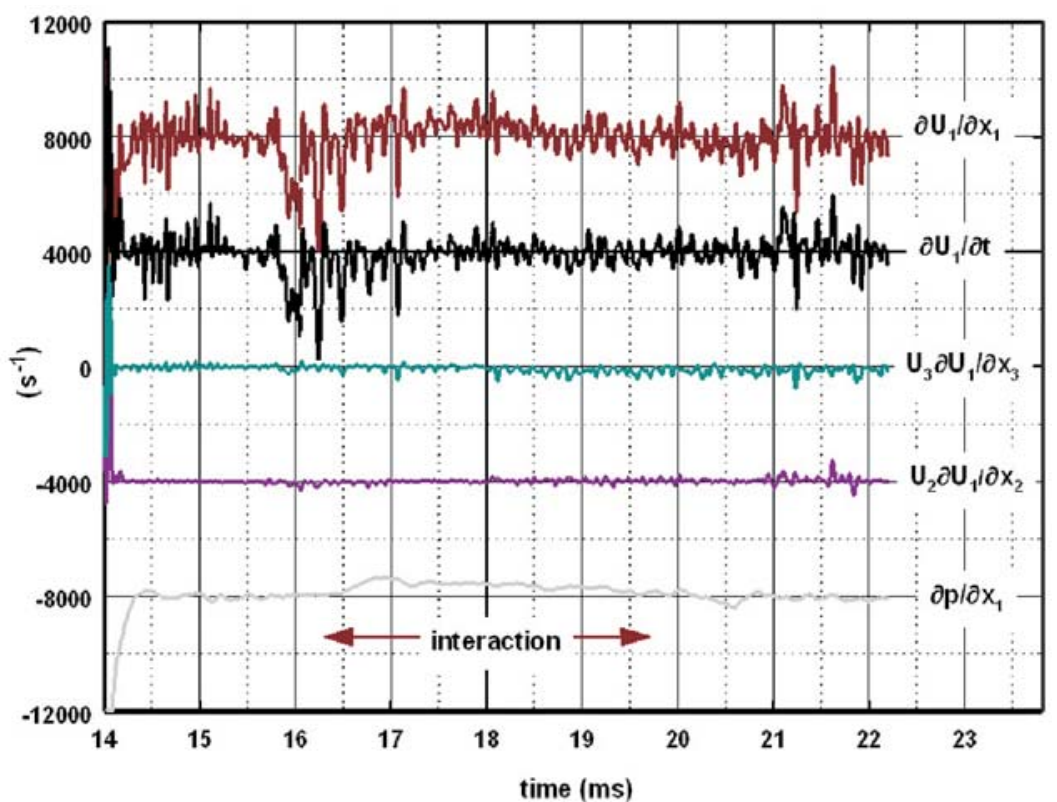

Fig. 7: Computation of velocity gradient, $\partial U_{1} / \partial x_{1}$, from contributing terms. Signals are shifted by multiples of $4,000 \mathrm{~s}^{-1}$.

the exception of the pressure gradients. Mean values of pressure gradients in the lateral directions are zero and their fluctuations are extremely small. In this respect, $\partial p / \partial x_{2}$ and $\partial p / \partial x_{3}$ have been neglected from the corresponding equations. The pressure gradient, $\partial p / \partial x_{1}$, which appears in the streamwise gradient of the longitudinal velocity is not zero through the flow expansion zone. Fig. 6 shows three wall pressure signals obtained at three different locations in the working section below the hot-wire probes. Their temporal gradients, $\partial p / \partial t$, are also shown in the same figure. These temporal gradients were converted to spatial gradients of pressure, $\partial p / \partial x_{1}$, by considering their propagation into the upstream flow, which takes place with a relative velocity equal to that of the local speed of sound, $C$. This propagation velocity of the EW relative to laboratory coordinates is $C(t)-U_{1}(t)$ where $U_{1}$ is the local flow velocity. Both time-dependent quantities, $C$ and $U_{2}$, are available from the combined temperature and velocity measurements. In this respect the spatial gradient was evaluated from the temporal gradient through the relation

$$
\frac{\partial p}{\partial x_{1}}=-\frac{\partial p}{\partial t} \frac{1}{C-U_{1}} .
$$

The contribution of this term as well as those of the other terms into the final computed value of $S_{11}=\partial U_{1} / \partial x_{1}$ is demonstrated in Fig. 7, where each of the individual terms is plotted separately. The data in Fig. 7 clearly show that the major contribution to $S_{11}$ comes from the term

$$
-\frac{1}{U_{1}} \frac{\partial U_{1}}{\partial t}
$$

which provides most of its high frequency content. The terms

$$
-\frac{U_{2}}{U_{1}} \frac{\partial U_{1}}{\partial x_{2}} \text { and }-\frac{U_{3}}{U_{1}} \frac{\partial U_{1}}{\partial x_{3}}
$$

are substantially smaller than the leading term a finding which also agrees with our previous work $[8,9]$. The contribution from the pressure gradient term

$$
\frac{1}{\rho U_{1}} \frac{\partial p}{\partial x_{2}}=-\frac{1}{\rho U_{1}} \frac{\partial p}{\partial t} \frac{1}{C-U_{1}}
$$

appears to provide a low frequency content of modest amplitude into the final value of $\partial U_{1} / \partial x_{1}$. The only assumption made in the present analysis is that the magnitude of the pressure fluctuations inside the flow field is negligible to the mean pressure upstream and downstream of the interaction or to the slowly varying pressure within the expansion zone. This assumption introduced an uncertainty into the computations which will be considered in the next section. It should be emphasized that only $S_{11}$ is affected directly by the pressure gradient along the expansion zone where the interaction takes place. The rest of the velocity gradients and therefore all components of vorticity, are not affected directly by pressure gradients.

\section{Uncertainty estimates}

The pressure and total temperature measurements depend directly, through the obtained calibration constants, on the raw voltage data from the individual sensors. Because of their linear response these probes produced two calibration constants, sensitivity and D.C. offset. Therefore estimates of the uncertainty in the measurements of pressure and total temperature acquired through a 16-bit A/D converter depended mostly on the bit resolution and the residual errors from the calibration constants. Uncertainties in the range of less than $0.5 \%$ in pressure and about $2 \%$ in total temperature were found for typical measurements of these two quantities.

The mass flux measurements were tied to significantly more complex relations, which depended on the individual and relative geometry of different sensors. Mass flux was found to depend on the following variables: captured raw voltage $E_{i}$, reference temperature $T_{\mathrm{r}}$, total temperature $T_{0}$, wire temperature $T_{\mathrm{w}}$, calibration constants and yaw or pitch coefficients. Uncertainty values for the velocity were estimated to be between 1-3\%. These values are slightly better than those in [8] and [9], because of higher resolution of the ADCs. In obtaining all these estimates the square root of 
the squares of all partial uncertainties involved was assumed to model the error propagation into the final results. A MATHCAD code was used to calculate the partial uncertainties. The density variation across the sensing area has also been estimated from the $\Delta p / p$ obtained above and the measured variation of total temperature $T_{0}$ through the uncertainty propagation formula:

$$
\frac{\Delta \rho}{\rho_{0}}=\left\{\left[\frac{\Delta p}{p_{T}}\right]^{2}+\left[\frac{\Delta T}{T_{0}}\right]^{2}\right\}^{\frac{1}{2}} .
$$

This predicted approximately the same uncertainty as in the case of the pressure variation. Following the work of Agui et al. [13], estimates of the uncertainties associated with the measurements of velocity gradients were also obtained by considering the propagation of the uncertainties in the measurement of each quantity involved in the process. A typical velocity gradient is measured through the following approximation:

$$
\frac{\partial U_{i}}{\partial x_{j}} \approx \frac{U_{2}-U_{1}}{l_{p}}=F,
$$

where $U_{2}$ and $U_{1}$ are the velocities at two nearby locations, $l_{p}$ is the distance between these locations. If the uncertainties in the measurements of $U_{2}$ and $U_{1}$ are the same, $\Delta U_{1}=\Delta U_{2}=\Delta U_{3}$, and $l_{p}$ is determined accurately, then the relative uncertainty $\Delta F / F$ will be given by:

$$
\frac{\Delta F}{F}=\left\{2\left[\frac{\Delta U}{U_{2}-U_{1}}\right]^{2}\right\}^{\frac{1}{2}} .
$$

A typical $U$ is $2 \%$ of mean $U$, which corresponds to about $2 \mathrm{~m} / \mathrm{s}$, while typical velocity differences $U_{2}-U_{1}$ can be up to six times the r.m.s. value, $u^{\prime}$. If a typical value of this velocity difference is assumed of about $30 \mathrm{~m} / \mathrm{s}$ in the near field of the grid and $15 \mathrm{~m} / \mathrm{s}$ further downstream, then the uncertainty $\mathrm{F} / \mathrm{F}$ appears to be $10 \%$ in the near field and $14 \%$ in the far field. Lower uncertainty estimates have been found if the relation

$$
\frac{\partial U_{i}}{\partial x_{j}} \approx \frac{u^{\prime}}{\lambda}=F
$$

is used for their computation. In this case the relative error is:

$$
\frac{\Delta F}{F}=\left\{\left[\frac{\Delta u^{\prime}}{u^{\prime}}\right]^{2}+\left[\frac{\Delta \lambda}{\lambda}\right]^{2}\right\}^{\frac{1}{2}},
$$

where $\lambda$ is Taylor's microscale. For a typical relative error in $u^{\prime}$ of $5 \%$ and $10 \%$ in $\lambda$ the relative error appears to be about $11 \%$. It should be noted that the relative error $F / F$ increases as the distance away from the grid increases because the absolute value of $F$ decreases.

Finally the finite number of statistically independent events considered in the data analysis of certain flow cases introduces an uncertainty in the statistical results. Computations of the integral time scale, $L_{t}$ from auto-correlation functions $R_{u u}(\tau)$ indicated that the number of independent samples in general was between 200 to 400 . Downstream of the interaction time scales $L_{t}$ increase and the number of statistically independent events is reduced. In addition, the duration of the useful data upstream of the shock is shortened at loca- tions close to the porous end wall because the reflected shock wave arrives earlier than at locations close to the grid. The onset of the useful data duration in the upstream of the shock region is also delayed by the arrival of the air mass has not gone through the grid. The number of independent samples in these cases was about $N \approx 60-100$. The relative error in the estimate of the variance of the velocity fluctuations is $2 / N$, which for this specific case at large $x / M$ is between 2 to $4 \%$. It should be noted that $N$ depends on the shape of $R_{u u}$, which can be extended to large values if low frequency disturbances are present in the flow field, which are not related to the actual flow turbulence. If high pass filtering at $200-400 \mathrm{~Hz}$ is applied to the present data $L_{t}$ is reduced substantially and $N$ increases by a factor of 2 . No such filtering has been applied to the present data other than what is imposed by the record length. For $10 \mathrm{~ms}$ record length the lowest frequency of interest is about $100 \mathrm{~Hz}$.

Furhter direct evidence of the adequacy of the statistical samples can be provided by the rate of convergence of the various statistical quantities that are computed in the present data analysis. As was shown [8], estimates of the convergence uncertainties observed in the present analysis indicate an error of less than $3 \%$. This error is substantially less at higher Mach numbers and locations at closer to the grid. The spatial resolution of the probe is between $0.6 \lambda$ and $3 \lambda$ in the upstream of the shock region and $0.3 \lambda$ and $\lambda$ in the downstream region. The resolution expressed in Kolmogorov's viscous scale $\eta=\left(v^{3 / 4} / \varepsilon\right)^{1 / 4}$ appears to be in the range between $3 \eta$ and $30 \eta$. In this respect the expected attenuation of the measurement of vorticity r.m.s. due to limited spatial resolution is not very significant.

\section{Conclusions}

A custom-designed vorticity probe was used to measure for the first time the rate-of-strain, the rate-of-rotation and the velocity-gradient tensors in compressible turbulent flows. Testing and validation of the probe and its eventual use in the shock tube flow field were formidable tasks. The difficulties associated with the measurements of velocity gradients in non-isothermal flows have been discussed. Issues related to calibration, data analysis and spatial and temporal resolutions appeared to be the most challenging.

Reynolds numbers based on Taylor's microscale ranging from 180 to 210 have been achieved. The interactions have been investigated by measuring the three-dimensional velocity and vorticity vectors, the full velocity gradient and rate-of-strain tensors with instrumentation of high temporal and spatial resolution. This allowed estimates of dilatation, compressible dissipation and dilatational stretching to be obtained.

\section{Acknowledgments}

The financial support provided by NASA \& AFOSR is greatly acknowledged.

\section{References}

[1] Lighthil, M. J.: Boundary Layer Theory in Laminar Boundary Layers, (ed. Rosenhead) Oxford University Press, (1963). 
[2] Agui, J. H., Andreopoulos, J.: Development of a New LASER Vorticity Probe-LAVOR. Fluids Engineering Division of ASME, International Symposium on LASER Anemometry, (eds. Huang, Otugen, Lake Tahoe NV), FED Vol. 191 (1994), p. 11-19, June 20-24, 1994.

[3] Agui, J. H, Andreopoulos, Y.: A New Laser Vorticity Probe - LAVOR: its Development and Validation in a Turbulent Boundary Layer. Experiments in Fluids, Vol. 34 (2003), p. 192-205.

[4] Hoknan, A., Andreopoulos, Y.: Vorticity, Strain-Rate and Dissipation Characteristics in the Near-Wall Region of Turbulent Boundary Layers. J. Fluid Mech. Vol. 350 (1997a), p. 29-96.

[5] Andreopoulos, Y, Honkan, A.: An Experimental Study of the Dissipative and Vortical Motions in Turbulent Boundary Layers. J. Fluid Mech. Vol. 439 (2001), p. 131-163.

[6] Hoknan, A., Andreopoulos, Y.: Instantaneous Three Dimensional Vorticity Measurements in Vortical Flow over a Delta Wing. AIAA J. Vol. 35 (1997b), No. 10, p. 1612-1620.

[7] Andreopoulos, Y., Agui, J. H, Briassulis, G.: Shock Wave-Turbulence Interactions. Annual Review Fluid Mech. Vol. 32 (2000), p. 309-345.

[8] Briassulis, G., Agui, J., Andreopoulos, Y.: The Structure of Weakly Compressible Grid Turbulence. J. Fluid Mech. Vol. 432 (2001), p. 219-283.

[9] Agui, J. H., Briassulis, G., Andreopoulos, Y.: Studies of Interactions of a Propagating Shock Wave with Decaying Grid Turbulence: Velocity and Vorticity Field. J. Fluid Mech. Vol. 524 (2005), p. 143-195.

[10] Vukoslavcevic, P., Wallace, J. M., Balint, J.: The Velocity and Vorticity Vector Fields of a Turbulent Boundary
Layer. Part 1 Simultaneous Measurement by Hot-wire Anemometry. J. Fluid Mech. Vol. 228 (1991), p. 25-51.

[11] Wallace, J. M., Foss, J.: The Measurement of Vorticity in Turbulent Flows. Annual Review Fluid Mech. Vol. 27 (1995), p. 469-514.

[12] Briassulis, G. K.: Unsteady Nonlinear Interactions of Turbulence with Shock Waves. Ph.D. Thesis. City College of CUNY, 1996, New York.

[13] Agui, J.: Shock Wave Interactions With Turbulence and Vortices. Ph.D. Thesis, City College of CUNY, 1998, New York.

[14] Briassulis, G., Honkan, A., Andreopoulos, J., Watkins, C. B.: Application of Hot-Wire Anemometry in Shock-Tube Flows. Experiments in Fluids. Vol. 19 (1995), p. 29.

[15] Agui, J. H., Andreopoulos, Y.: A New Laser Vorticity Probe - LAVOR: its Development and Validation in a Turbulent Boundary Layer. Exp. Fluids. Vol. 34 (2003), No. 2, p. 192-205.

$\overline{\text { Savvas Xanthos, Ph. D. }}$

Minwei Gong, Ph.D.

Yiannis Andreopoulos, Ph.D.

Experimental Aerodynamics and Fluid Mechanics Laboratory

The Mechanical Engineering Department The City College of the City University of New York New York, New York 10031 USA 